\begin{tabular}{|c|c|}
\hline Title & Multigrid Method With A daptive IDR-Based Jacobi Smoother \\
\hline Author(s) & W atanabe, Kota; Fujino, Seiji; Igarashi, Hajime \\
\hline Citation & $\begin{array}{l}\text { IEEE T ransactions on Magnetics, 47(5), 1210-1213 } \\
\text { https://doi.org/10.1109/ MA G.2010.2092754 }\end{array}$ \\
\hline Issue Date & $2011-05$ \\
\hline Doc URL & http:/hdl.handle.net/2115/46204 \\
\hline Rights & $\begin{array}{l}\text { (c) } 2011 \text { IEEE. Personal use of this material is permitted. Permission from IEEE must be obtained for all other uses, in } \\
\text { any current or future media, including reprinting/republishing this material for advertising or promotional purposes, } \\
\text { creating new collective works, for resale or redistribution to servers or lists, or reuse of any copyrighted component of } \\
\text { this work in other works. }\end{array}$ \\
\hline Tyре & article (author version) \\
\hline File Information & ToM 47-5_1210-1213.pdf \\
\hline
\end{tabular}

Instructions for use 


\title{
Multigrid Method with Adaptive IDR-based Jacobi Smoother
}

\author{
Kota Watanabe ${ }^{1}$, Seiji Fujino ${ }^{2}$ and Hajime Igarashi ${ }^{1}$, Member, IEEE \\ ${ }^{1}$ Graduate School of Information and Technology, Hokkaido University, Sapporo 060-0814, Japan \\ ${ }^{2}$ Research Institute for Information Technology, Kyushu University, Fukuoka 812-8581, Japan
}

\begin{abstract}
Multigrid (MG) methods are known to be fast linear solvers for large-scale finite element analyses. The Gauss-Seidel method is usually adopted as the smoother for MG methods. However, recently, considerable attention has been focused on Induced Dimension Reduction (IDR)-based solvers because they are faster. In this paper, we investigate the convergence of IDR-based solvers and evaluate the performance of a MG method with an adaptive IDR-based Jacobi smoother. Numerical results show that this method has good convergence and good efficiency in parallel computations for finite element analysis of electromagnetic fields.
\end{abstract}

Index Terms-Finite element method, Induced dimension reduction method, Multigrid method, Parallel computation

\section{INTRODUCTION}

$\mathrm{M}$ ULTIGRID (MG) methods have been shown to be faster in solving electromagnetic field problems[1]. In the finite element analysis of electromagnetic fields, the simple or hybrid Gauss-Seidel method is usually adopted as the smoother for both algebraic and geometric MG methods [2]-[4]. The reason for this wide adoption of the Gauss-Seidel method is that at the start of the iterative process, highfrequency components of residues are more rapidly reduced than the low-frequency components. However, the GaussSeidel method itself converges slowly and moreover, is not suitable for parallel computations, although the use of some parallel algorithms such as the multi-color ordering method with shared memory systems is well known [5].

The Induced Dimension Reduction (IDR) method proposed by Sonneveld et al. in 1980 is an iterative solver based on the IDR theorem [6]. However, this method has not been used as a standard solver for these 30 years. In 2008, Sonneveld and van Gijzen proposed the IDR(s) method, which is an extension of the IDR method [7]. Lately, the IDR(s) method and a family of IDR(s) have attracted considerable attention for solving nonsymmetric system of equations, because these methods are faster than the present state-of-the-art solvers such as BiCGStab and GMRESS(k) [8]. In particular, the adaptive IDR-based Jacobi method has good convergence for the symmetric systems obtained from finite element analyses as well as for nonsymmetric systems of equations. Moreover, because this method involves matrix-vector multiplications and vector inner-products, it has high parallel efficiency on distributed memory as well as shared memory systems. In this paper, we investigate the convergence profile of the IDR(s) family of solvers and present the performance of a $\mathrm{MG}$ method coupled the adaptive IDR-based Jacobi smoother in static magnetic field analyses.

\section{FORMULATION}

\section{A. Multigrid method}

Considering the magnetic vector potential, the differential

Manuscript received May 31, 2010. Corresponding author: K. Watanabe (watanabe@ssi.ist.hokudai.ac.jp).

Digital Object Identifier inserted by IEEE equation for the magnetostatic field is

I.

$\nabla \times \frac{1}{\mu} \nabla \times \boldsymbol{A}=\boldsymbol{J}_{0}$,

where $\mu$ is the permeability, $\boldsymbol{J}_{0}$ is the given current density, and $\boldsymbol{A}$ is the magnetic vector potential. Finite element discretization of (1) on a fine mesh results in the following system of linear equations:

$\mathrm{K}^{f} \boldsymbol{x}=\boldsymbol{b}$,

where $\mathrm{K}^{f}$ is a system matrix on the fine mesh.

It is known that linear solvers such as the Gauss-Seidel method tend to eliminate the high-frequency residual components in (2) more rapidly than the low-frequency ones. The MG method is based on this property; that is, the highfrequency residual components are eliminated on a fine mesh within a few iterations of the linear solver (smoother). The remaining residual components are then projected onto a coarser mesh, where those with a higher frequency can again be eliminated within a few iterations. The MG method solves (2) by iterating the abovementioned process. The two-grid Vcycle MG method, which is the simplest MG method, is described below.

Step1: (Smoothing): The smoothing is applied to $\mathrm{K}^{f} \boldsymbol{x}=\boldsymbol{b}$, and an approximated solution $\tilde{\boldsymbol{x}}$ is obtained.

Step2: (Restriction) $\boldsymbol{r}^{c}=\mathrm{R} \boldsymbol{r}^{f}$, where $\boldsymbol{r}^{f}=\boldsymbol{b}-\mathrm{K}^{f} \tilde{\boldsymbol{x}}$.

Step3: Solve $\mathrm{K}^{c} \boldsymbol{e}^{c}=\boldsymbol{r}^{c}$ to obtain the error vector $\boldsymbol{e}^{c}$.

Step4: (Prolongation): $\boldsymbol{e}^{f}=\mathrm{R}^{t} \boldsymbol{e}^{c}$.

Step5: $\tilde{\boldsymbol{x}} \leftarrow \tilde{\boldsymbol{x}}+\boldsymbol{e}^{f}$

Step6: (Post-Smoothing): The smoothing is applied again and the convergence of the solution is tested. If the convergence condition is not satisfied, the process is repeated from Step2 onwards.

In the nested MG method, we can construct the restriction matrix R using the geometric relationship between the coarse and fine edges [9]-[10]. 


\section{B. IDR(s)-based methods}

The Jacobi method is known as a classical simple linear solver and it shows slow convergence or divergence of iterations for several problem types. For this reason, this method is not used for solving practical problems. By contrast the IDR(s)-based Jacobi method, which is based on the IDR theorem described as follows, shows good convergence. Let $\mathrm{A} \in R^{N \times N}$ be any matrix, $\boldsymbol{v}_{0} \in R^{N}$ be any vector, and $\vartheta_{0}$ be the complete Krylov space $K_{N}\left(\mathrm{~A}, v_{0}\right)$. Let $\mathrm{S}$ denote any subspace of $R^{N}$ such that $\mathrm{S} \cap \vartheta_{0} \notin \mathrm{A}$ and then, we define the sequence spaces $\vartheta_{i}(i=1,2, \ldots)$ as,

$$
\vartheta_{i} \equiv\left(\mathrm{I}-\omega_{i} \mathrm{~A}\right)\left(\vartheta_{i-1} \cap \mathrm{S}\right),
$$

where $\omega_{i}$ are nonzero scalars and I is the identity matrix. Then, the following two theorems hold:

$$
\begin{aligned}
& \vartheta_{i} \subseteq \vartheta_{i-1} \quad \text { for all } \quad i>0 \\
& \vartheta_{i}=0 \text { for some } i \leq N
\end{aligned} .
$$

If the $i$-th residual vector $\boldsymbol{r}_{i}$ is in the space $\vartheta_{i}$, the size of the space decreases monotonously and then converges to zero. For this purpose, the residual vector should satisfy

$$
\boldsymbol{r}_{i+1} \equiv\left(\mathrm{I}-\omega_{i+1} \mathrm{~A}\right) \boldsymbol{v},
$$

where $\boldsymbol{v}$ consists of residual sequences $\left(\boldsymbol{r}_{i}, \boldsymbol{r}_{i-1}, . ., \boldsymbol{r}_{i-\mathrm{s}+1}\right)$

$$
\boldsymbol{v}=\boldsymbol{r}_{i}-\sum_{j=1}^{s} \gamma_{j} \boldsymbol{e}_{i-j},
$$

where $\boldsymbol{e}_{i} \equiv \boldsymbol{r}_{i}-\boldsymbol{r}_{i-1}$. Let $\mathrm{E}_{i}$ be the matrix $\left\{\boldsymbol{e}_{i}, \boldsymbol{e}_{i-1}, . ., \boldsymbol{e}_{i-\mathrm{s}+1}\right\}$ and vector $c$ that satisfy

$$
\mathrm{E}_{i} \boldsymbol{c}=\sum_{j=1}^{s} \gamma_{j} \boldsymbol{e}_{i-j} .
$$

Equation (6) is rewritten as $\boldsymbol{v}=\boldsymbol{r}_{i}-\mathrm{E}_{i} \boldsymbol{c}$. Moreover, considering any matrix $\mathrm{P}$ that satisfies $\mathrm{P}^{\mathrm{t}} \boldsymbol{v}=0$, we can obtain $\boldsymbol{c}$ by solving the equation

$$
\mathrm{P}^{\mathrm{t}} \mathrm{E}_{i} \boldsymbol{c}=\mathrm{P}^{\mathrm{t}} \boldsymbol{r}_{i} .
$$

Vector $\boldsymbol{e}$ is calculated as

$$
\begin{aligned}
\boldsymbol{e}_{i} & =\boldsymbol{r}_{i}-\boldsymbol{r}_{i-1} \\
& =-\mathrm{E}_{i} \boldsymbol{c}-\omega_{i} \mathrm{~A} \boldsymbol{v} .
\end{aligned}
$$

The update vector for solution $q_{i} \equiv \boldsymbol{x}_{i}-\boldsymbol{x}_{i-1}$ is expressed as

$$
\boldsymbol{q}_{i}=-Q_{i} \boldsymbol{c}+Z_{i} \boldsymbol{v},
$$

where $\mathrm{Q}_{i}$ is a matrix $\left\{\boldsymbol{q}_{i}, \boldsymbol{q}_{i-1}, . ., \boldsymbol{q}_{i-\mathrm{s}+1}\right\}$.

The IDR(s) method is based on the above equations. Note that system matrix is not assumed to be symmetrical. Therefore, the IDR(s) method can be applied to nonsymmetric system as well as symmetric system equations.

The IDR(s)-based Jacobi (I-Jacobi), Gauss-Seidel (I-GS) and Successive Over-Relaxation (I-SOR) methods were proposed by an author of this paper in 2009 [8]; the algorithms are shown in Fig. 1. In this figure, the matrix $\mathrm{N}$ and $\mathrm{M}$ are defined as $\mathrm{A} \equiv \mathrm{M}-\mathrm{N}$ and selected as

I-Jacobi: $\mathrm{M}=\mathrm{D} / \omega, \mathrm{N}=\mathrm{A}-\mathrm{D} / \omega$

I-GS $: \mathrm{M}=(\mathrm{L}+\mathrm{D})^{-1}, \mathrm{~N}=\mathrm{U}$

I-SOR : $\mathrm{M}=(\mathrm{L}+\mathrm{D} / \omega)^{-1}, \mathrm{~N}=\left(\mathrm{I}-\omega^{-1}\right) \mathrm{D}+\mathrm{U}$,

where $\mathrm{D}$ is the diagonal, $\mathrm{L}$ is the strictly lower triangular, and $\mathrm{U}$ is the strictly upper triangular matrix of A. In I-Jacobi, the parameter $\omega$ is either defined by the user or obtained adaptively to minimize the norm of $\boldsymbol{r}_{i+1}$

$\omega_{i}=\left(\mathrm{D}^{-1} \mathrm{~A} \boldsymbol{t}_{i}, \boldsymbol{t}_{i}\right) /\left(\mathrm{D}^{-1} \mathrm{~A} \boldsymbol{t}_{i}, \mathrm{~A} \boldsymbol{t}_{i}\right)$,

where $\boldsymbol{t}_{i}=\boldsymbol{r}_{i}-\mathrm{E} \boldsymbol{c}$. The matrix $\mathrm{P}$ can be arrived at by applying Gramm-Schmidt orthonormalization to the matrix $\left\{\boldsymbol{r}_{0}, *\right.$, $*, \ldots, *\} \quad(*$ denotes random vectors).

We named the combination of (11) and I-Jacobi as the "adaptive IDR(s) based Jacobi" method.

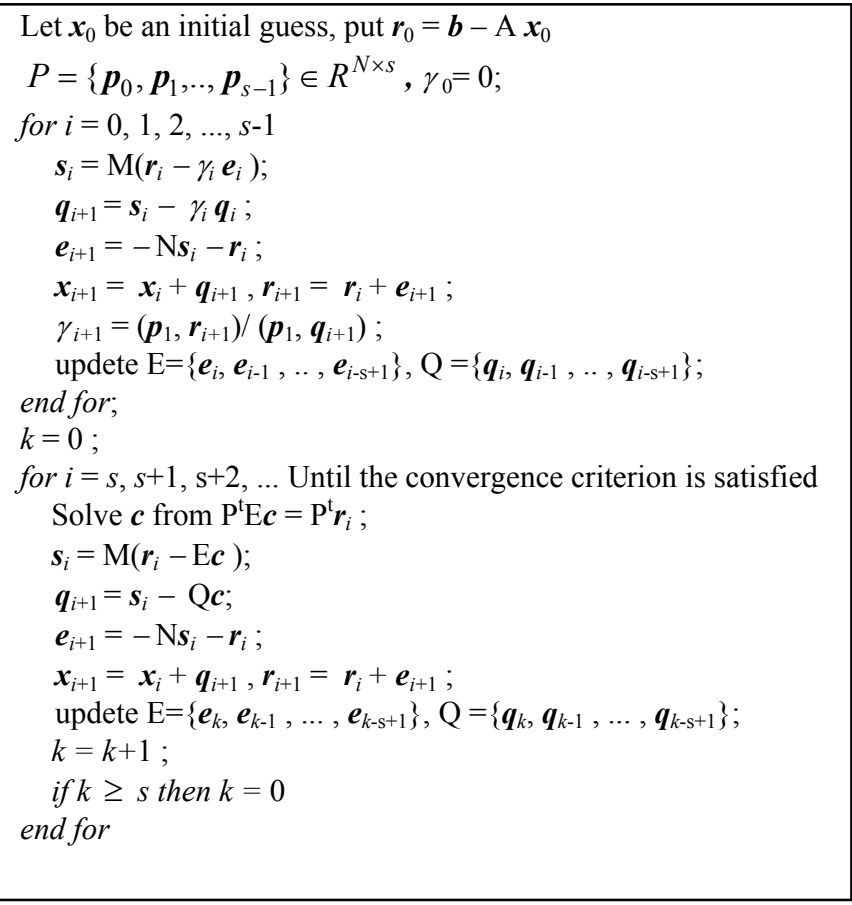

Fig. 1. Algorithm of IDR(s)-based method

\section{NUMERICAL RESULTS}

\section{A. Comparison of solvers}

First, we evaluated the convergence of the system equations obtained from the analysis of a magnetostatic field with tetrahedral edge elements, as shown in Fig. 2. Fig. 3 shows the convergence profile obtained using various solvers: classical Jacobi, Gauss-Seidel (GS), ICCG, IDR(s), and the adaptive IJacobi method. We obtained the following numerical results. The classical Jacobi method does not converge and the residual norm increases linearly. By contrast, the ICCG method shows good convergence. Except at the beginning of 
the iterative process, the GS method shows very slow convergence. The IDR(s) and adaptive I-Jacobi methods also shows slow convergence with some sharp peaks; IDR(5) and adaptive I-Jacobi(3), in particular, oscillate randomly. Identical convergence profiles are observed in other cases for $\mathrm{s}$ $>1$. In our experience, the convergence of IDR(s)-based methods improves with an increase in s; however, this improvement is not observed in the preliminary stages of the iterative process. For the above reason and increased computational costs for $\mathrm{s}>1$, $\mathrm{s}$ should be unity for a $\mathrm{MG}$ smoother.

Next, we investigate the capability of the smoother in IDR(s) methods. It is important that smoothers rapidly decrease the high-frequency components of residue. Thus, we investigate the components of reduction rate after 50 smoothing iterations.

$\gamma_{50}^{k}=\left|\left(\boldsymbol{r}_{50}, \boldsymbol{v}_{k}\right)\right| /\left|\left(\boldsymbol{r}_{0}, \boldsymbol{v}_{k}\right)\right| k=1,2, \ldots N_{e}$

where $\boldsymbol{v}_{k}$ is the eigenvector corresponding to the $k$-th non-zero eigenvalue and $N_{e}$ is the number of non-zero eigenvalues. Fig. 4 shows the components that are the average of 10 trials, each beginning with a random initial guess $\boldsymbol{x}_{0}$. We note that the eigenvector corresponding to the higher-order eigenvalue in Fig. 4 represents a higher-frequency distribution. It is also apparent that the GS and adaptive I-Jacobi methods eliminate the high-frequency components faster than the low-frequency components. Therefore, the adaptive I-Jacobi method can be used as the smoother.
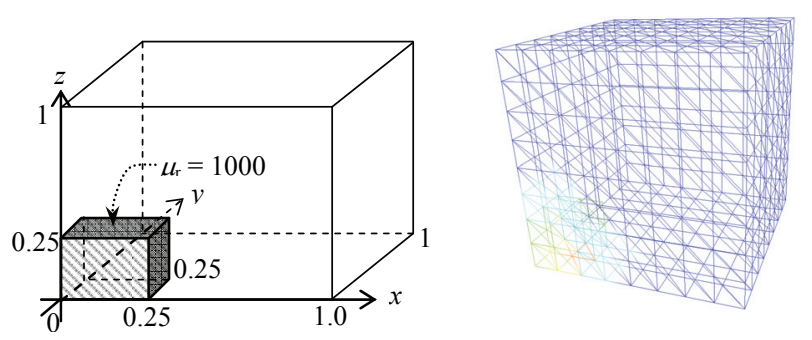

Fig. 2 Magnetostatic model for checking convergence of solvers. Number of unknowns is 7768 (coarse mesh). An external constant magnetic field is applied along the z-direction to the entire region.

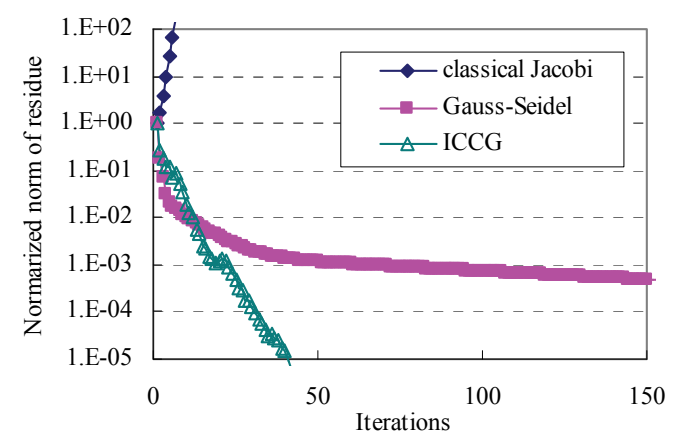

(a)

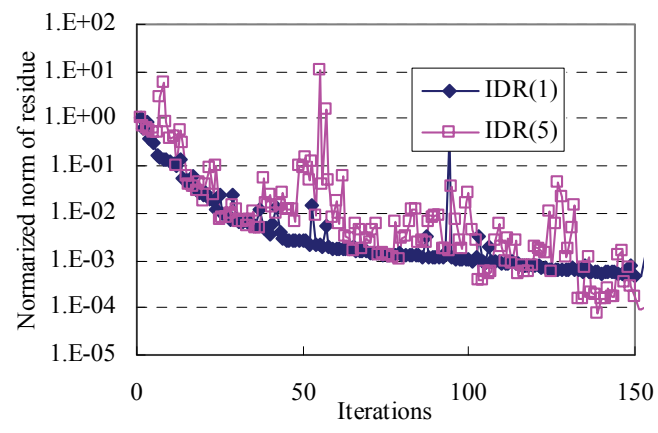

(b)

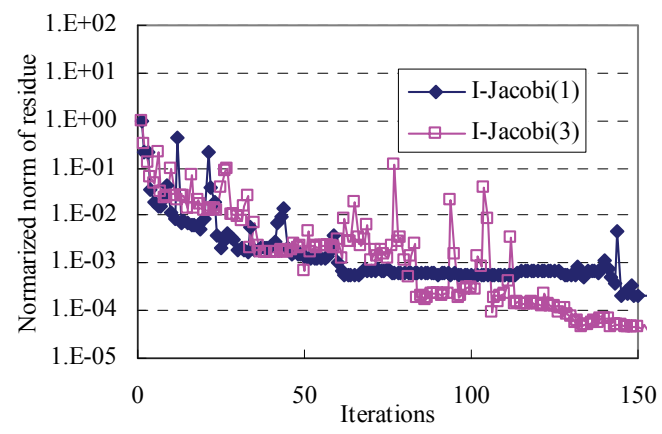

(c)

Fig. 3 Normalized residual norm history of various solvers, (a) Conventional solvers, (b) IDR solver, (c) Adaptive IDR(s)-Jacobi method

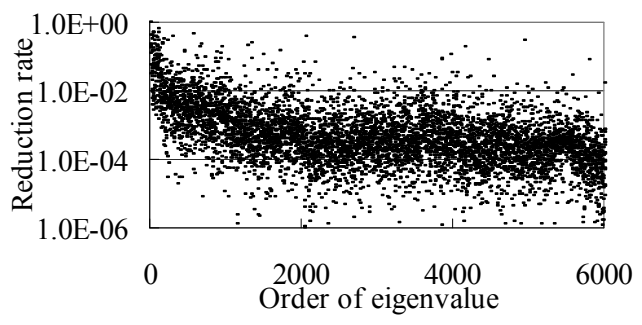

(a)

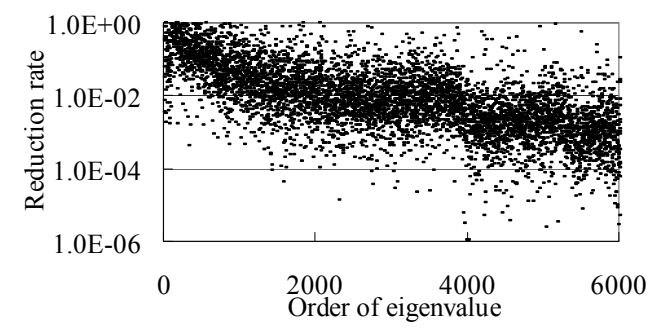

(b)

Fig. 4 Components of reduction rate sorted in ascending order (a) Gauss-Seidel, (b) Adaptive I-Jacobi.

\section{B. Results of $M G$ method}

Finally, we apply the adaptive IDR(1)-based Jacobi (IJacobi) method to a MG. We analyze the simple model shown in Fig. 2 and the perpendicular magnetic recording head model shown in Fig 5 [10]. The convergence criterion is $|\boldsymbol{r}|<10^{-6}|\boldsymbol{b}|$. Table I compares the convergences and computational times of a 2-grid MG of the magnetic head model using the GS smoother and the adaptive I-Jacobi smoother. The computations were performed on a PC with two CPUs (Intel Xeon 5160). Parallel computation using Open 
MP with 4 threads was used.

We can infer from Table I that the adaptive I-Jacobi smoother requires a fewer number of iterations and lesser computational time to converge as compared to the GS smoother. The effects of parallel computation on the 3-grid MG of the model shown in Fig. 2 are summarized in Table II. The adaptive I-Jacobi procedures for $\mathrm{s}=1$ consist of matrixvector multiplications and vector inner-products and these operations have high parallel efficiency.

A comparison between the MG and the MG preconditioned conjugate gradient (CG) method is shown in Table III. The GS smoother works well for both methods. On the other hand, the MG preconditioned CG in conjunction with the adaptive IJacobi smoother is slower than that in conjunction with the GS smoother. The reason for this will be investigated in further work.

Finally, Fig. 6 shows the convergence profile for 40 smoothing/V-cycle entry, listed in Table I. The MG in conjunction with the adaptive I-Jacobi smoother shows some peaks; however, the overall convergence achieved using this smoother is better than that achieved using the GS smoother.

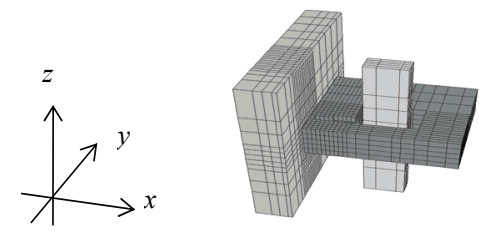

Fig. 5 Model of magnetic recording head; hexahedral edge elements are used.

Table I. Convergence and computation time for a 2-grid MG of magnetic head model with 141,160 DoFs

\begin{tabular}{cccc}
\hline $\begin{array}{c}\text { Type of } \\
\text { smoother }\end{array}$ & $\begin{array}{c}\text { Number of } \\
\text { smoothing/V cycle }\end{array}$ & $\begin{array}{c}\text { Number of } \\
\text { V-cycles }\end{array}$ & Elapsed time $[\mathrm{s}]$ \\
\hline \multirow{2}{*}{ Gauss- } & 30 & 12 & 18 \\
Seidel & 40 & 9 & 17 \\
& 50 & 8 & 19 \\
\hline \multirow{2}{*}{ Adaptive } & 30 & 9 & 13 \\
I-Jacobi & 40 & 6 & 11 \\
& 50 & 5 & 12 \\
\hline
\end{tabular}

Table II. Parallel efficiency for the 3 -grid MG of the simple model with 468,064 DoFs.

\begin{tabular}{cccc} 
& \multicolumn{2}{c}{ with 468,064 DoFs. } \\
\cline { 3 - 4 } Number of & Number & \multicolumn{2}{c}{ Elapsed time [s] } \\
\cline { 3 - 4 } smoothing /V cycle & of V- & without Open MP & using Open MP \\
\hline 30 & 8 & 55 & 23 \\
40 & 5 & 48 & 20 \\
50 & 5 & 60 & 24 \\
\hline
\end{tabular}

Table III. Comparison of standard MG and CG with MG preconditioner for a simple model with 468,064 DoFs

\begin{tabular}{ccccc}
\hline Method & $\begin{array}{c}\text { Type of } \\
\text { smoother }\end{array}$ & $\begin{array}{c}\text { Optimal } \\
\text { number of } \\
\text { smoothing } \\
\text { /V cycle }\end{array}$ & $\begin{array}{c}\text { Number of } \\
\text { V-cycles }\end{array}$ & $\begin{array}{c}\text { Elapsed } \\
\text { time [s] }\end{array}$ \\
\hline MG & GS & 20 & 8 & 13 \\
\hline MG & I-Jacobi & 40 & 5 & 20 \\
\hline $\begin{array}{c}\text { preconditioned } \\
\text { CG }\end{array}$ & GS & 15 & 17 & 11 \\
\hline
\end{tabular}

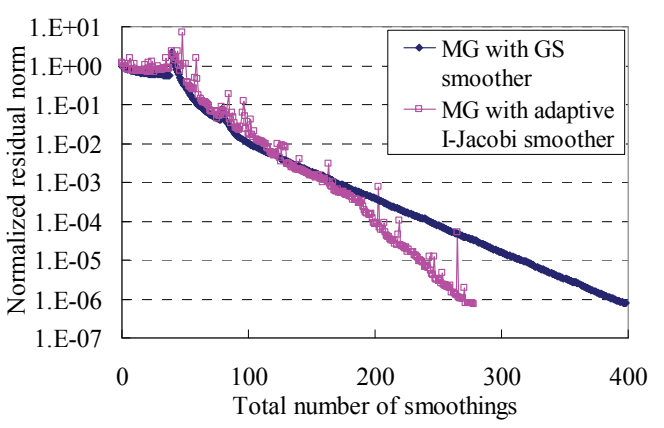

Fig. 6 Normalized residual norm history for MG

\section{CONCLUSION}

We evaluated the use of a multigrid method in conjunction with IDR-based smoothers for finite element analysis of electromagnetic fields. Although the IDR-based methods show unstable convergence, the numerical results show that the multigrid with adaptive IDR-based Jacobi smoother converges well in static magnetic field analyses. Moreover, the present method has better parallel efficiency that the Gauss-Seidel smoother. As such, the present method would perform well on distributed memory environments such as PC clusters.

\section{ACKNOWLEDGMENT}

This work was supported by a Grant-in-Aid for Scientific Research (21710165).

\section{REFERENCES}

[1] R. Hiptmair, "Multigrid method for Maxwell's equations," SIAM $J$. Numerical Analysis, Vol.36, pp.204-225, 1998.

[2] D. Arnold, R. Falk, and R. Winther, "Multigrid in H(div) and H(curl)," Numer. Math., Vol. 85, pp. 197-218, 2000.

[3] B. Weiß and O. Bíró, "Smoothing Operators for Edge Element Multigrid," IEEE Trans. on Magn., Vol. 38, No. 2, pp. 397-400, 2002.

[4] T. Mifune, T. Iwashita and M. Shimasaki, "New algebraic multigrid preconditioning for iterative solvers in electromagnetic finite edgeelement analyses," IEEE Trans. on Magn., Vol. 39, No. 3, pp. 1677$1680,2003$.

[5] L. Adams and J. Ortega, "A multi-color SOR method for parallel computation," Proc. of the 1982 International Conference on Parallel Processing, pp. 53-56, 1982.

[6] P. Wesseling and P. Sonneveld, "Numerical Experiments with a Multiple Grid and a Preconditioned Lanczos Type Method," Lecture Notes in Mathematics, Springer Berlin, Heidelberg, New York, Vol. 771, pp. 543-562, 1980.

[7] P. Sonneveld, M.B. van Gijzen, "IDR(s): A Family of Simple and Fast Algorithms for Solving Large Nonsymmetric Systems of Linear Equations," SIAM J. Sci. Comput. Vol. 31, No. 2, pp. 1035-1062, 2008.

[8] Y. Kusakabe, S. Fujino, "A proposal of Jacobi method based on extended Induced Dimension Reduction Theorem aimed at high convergence rate," The Proc. of EMAC 2009, Adelaide, 6-9, December, 2009.

[9] M Schinnerl, J. Schoberl, M. Kaltenbacher, "Nested multigrid methods for the fast numerical computation of 3D magnetic fields," IEEE Trans. Magn., Vol. 36, No. 4, pp. 1557-1560, 2000.

[10] K. Watanabe and H. Igarashi, "Robustness of Nested Multigrid Method for Edge-Based Finite Element Analysis," IEEE Trans. Magn., Vol. 45, No. 3, pp. 1088-1091, 2009. 